\title{
LIGOPHORUS PILENGAS N. SP. (MONOGENEA: ANCYROCEPHALIDAE) FROM THE INTRODUCED SO-IUY MULLET, MUGIL SOIUY (TELEOSTEI: MUGILIDAE), IN THE SEA OF AZOV AND THE BLACK SEA
}

\author{
Volodimir Leonidovich Sarabeev and Juan Antonio Balbuena* $†$ \\ Department of Biology, Zaporizhzhia State University, 66 Zhukovskogo Street, 69063 Zhaporizhzhia, Ukraine. e-mail: j.a.balbuena@uv.es
}

\begin{abstract}
The monogenean Ligophorus chabaudi was originally described on the gills of the flathead mullet, Mugil cephalus, and was subsequently reported on the So-iuy mullet, Mugil soiuy. However, the morphology of sclerotized parts and multivariate statistical analyses suggest that the form from the So-iuy mullet represents a new species. This study provides a description of the new species Ligophorus pilengas $\mathrm{n}$. sp. and provides additional morphological data concerning the morphology of the ventral bar that might be useful for the diagnosis of Ligophorus. Ligophorus pilengas $\mathrm{n}$. $\mathrm{sp}$. is the second species of Ligophorus reported on the So-iuy mullet. Zoogeographical records indicate that L. pilengas $\mathrm{n}$. sp. was probably introduced to the Black Sea and the Sea of Azov from the western Pacific Ocean together with its host.
\end{abstract}

Euzet and Suriano (1977) erected Ligophorus within the Ancyrocephalidae and included 11 species parasitizing the gills of mullets (Mugilidae) from the Mediterranean Sea and the North Atlantic: Ligophorus vanbenedenii (Parona and Perugia, 1890); Ligophorus szidati Euzet and Suriano, 1977; Ligophorus mugilinus (Hargis, 1955); Ligophorus chabaudi Euzet and Suriano, 1977; Ligophorus macrocolpos Euzet and Suriano, 1977; Ligophorus acuminatus Euzet and Suriano, 1977; Ligophorus minimus Euzet and Suriano, 1977; Ligophorus heteronchus Euzet and Suriano, 1977; Ligophorus angustus Euzet and Suriano, 1977; Ligophorus imitans Euzet and Suriano, 1977; and Ligophorus confusus Euzet and Suriano, 1977. Subsequently 9 new species have been added to the genus, expanding their distribution range to the North and South Pacific: Ligophorus leporinus (Zhang and Ji, 1981); Ligophorus parvicirrus Euzet and Sanfilippo, 1983; Ligophorus kaohsianghsieni (Gusev, 1962); Ligophorus huitrempe Fernández, 1987; Ligophorus chongmingensis $\mathrm{Hu}$ and $\mathrm{Li}, 1992$; Ligophorus chenzhenensis $\mathrm{Hu}$ and $\mathrm{Li}$, 1992; Ligophorus euzeti Dmitrieva and Gerasev, 1996; Ligophorus hamulosus Pan, 1999; and Ligophorus ellochelon Yang, 2001 (Euzet and Sanfilippo, 1983; Gusev, 1985; Dmitrieva and Gerasev, 1996; Zhang et al. 2003).

Euzet and Suriano (1977) considered that all species of Ligophorus are oioxenic, which seemed substantiated by ensuing studies (Euzet and Sanfilippo, 1983; Radujković and Raibaut, 1989; Caltran et al., 1995; Caillot et al., 1999). However, current records show that at least some species are stenoxenic. For instance, L. kaohsianghsieni, whose typical host is the So-iuy mullet, Mugil soiuy Basilewsky, has also been reported on the flathead mullet, Mugil cephalus L., and golden gray mullet, Liza aurata (Risso) (Dmitrieva, 1996; Miroshnichenko and Maltsev, 1998). Likewise, L. vanbenedenii, common on L. aurata, has also been recorded on $M$. cephalus, and L. macrocolpos, typical on the leaping mullet, Liza saliens (Risso), occurs also on $L$. aurata (Dmitrieva and Gerasev, 1996). However, the prevalence and abundance on such alternative hosts is much lower than those on the typical host of each species of Ligophorus. Apparently, the only exception to this pattern is $L$. chabaudi be-

Received 5 June 2003; revised 2 September 2003; accepted 3 September 2003.

* Marine Zoology Unit, Cavanilles Institute of Biodiversity and Evolutionary Biology, University of Valencia, P.O. Box 22085, 46071 Valencia, Spain.

$\dagger$ To whom correspondence should be addressed. cause it occurs typically on M. cephalus; however, according to previous studies and our own data, it also shows high prevalence (87-100\%) and abundance (up to 736 individuals) on adult So-iuy mullets (Dmitrieva, 1996; Sarabeev and Domnich, 2000).

In this article, we compared the morphology of haptoral and genital sclerotized parts and conducted a linear discriminant analysis of metrical features of putative $L$. chabaudi from $M$. cephalus and $M$. soiuy. The results suggest that the form from M. soiuy probably represents a new species, Ligophorus pilengas n. sp., which is described in this article.

\section{MATERIALS AND METHODS}

Mullets were collected from the Sea of Azov, the Black Sea, and the Western Mediterranean Sea (Table I). Fish were examined for parasites within the day of capture and surveyed for Ligophorus infection using a stereomicroscope. Eighty-five specimens belonging to 6 species of Ligophorus (Table I) were mounted in glycerin jelly, according to Gusev (1983), and measured. To study details of their internal anatomy, 15 additional specimens of $L$. pilengas $\mathrm{n}$. $\mathrm{sp}$. were stained in alum carmine, passed through a series of increasing ethanol concentrations (from 70 to $100 \%$ ), cleared in dimethyl phthalate, and mounted in Canada balsam.

Based on Gusev (1985) and Euzet and Suriano (1977), 19 characters were selected for morphometric analysis. The following abbreviations are used for the characters: BL, body length; BW, body width; VAA, ventral anchor total length (dorsoapical); VAB, ventral anchor main part length; VAC, ventral anchor outer root length; VAD, ventral anchor inner root length; VAE, ventral anchor point length; DAA, dorsal anchor total length (dorsoapical); DAB, dorsal anchor main part length; $\mathrm{DAC}$, dorsal anchor outer root length; DAD, dorsal anchor inner root length; DAE, dorsal anchor point length; HL, marginal hook total length; VBL, ventral bar length; VBAP, distance between membranous anterior processes of ventral bar; DBL, dorsal bar length; PAPL, penis accessorial piece length; PL, total length of penis; VL, vagina length. See Figure 1 for definition of measurements of sclerotized elements of haptors and genital structures.

All measurements of worms are given in micrometers as mean \pm standard deviation (range). Measurements and drawings were made using a Lomo P 15 microscope with phase contrast and a Nikon Optiphot2 microscope with interference contrast (magnification: $10 \times 10 \times$ for the body and $10 \times 100 \times 1.25 \times$ [under immersion oil] for sclerotized structures). Photographs of sclerotized structures of haptor and male copulative apparatus were taken with a Leica DMR microscope with interference contrast and a Leica DC300 camera (magnification of 10× $100 \times$ under immersion oil).

We used stepwise linear discriminant analysis to analyze the morphometric differences between the 85 specimens of the 6 Ligophorus species studied in this work (Table I). Initially, 15 of the 19 morphometric characters, namely, VAA, VAB, VAC, VAD, VAE, DAA, DAB, DAC, DAD, DAE, HL, VBL, VBAP, DBL, and PAPL, were chosen for 
TABLE I. Zoogeographical and host information on the specimens of Ligophorus used for morphometric analysis in this study.

\begin{tabular}{|c|c|c|c|c|c|}
\hline Parasite & Host & Locality* & Date & $\mathrm{n}$ & $\begin{array}{l}\text { Total length } \\
\text { of host (mm) }\end{array}$ \\
\hline Ligophorus pilengas $\mathrm{n}$. $\mathrm{sp}$. & Mugil soiuy & $\mathrm{ME}, 46^{\circ} 26^{\prime} \mathrm{N}, 35^{\circ} 26^{\prime} \mathrm{E}$ & June 2000 & 2 & 540 \\
\hline L. pilengas $\mathrm{n}$. $\mathrm{sp}$. & M. soiuy & $\mathrm{UE}, 46^{\circ} 21^{\prime} \mathrm{N}, 35^{\circ} 14^{\prime} \mathrm{E}$ & August 2002 & 12 & $332-510$ \\
\hline L. pilengas $\mathrm{n} . \mathrm{sp}$. & M. soiuy & $\mathrm{ME}, 46^{\circ} 26^{\prime} \mathrm{N}, 35^{\circ} 26^{\prime} \mathrm{E}$ & August 2002 & 8 & $470-515$ \\
\hline L. chabaudi & M. cephalus & $\mathrm{KC}, 45^{\circ} 20^{\prime} \mathrm{N}, 36^{\circ} 30^{\prime} \mathrm{E}$ & October 2001 & 15 & $315-390$ \\
\hline L. mugilinus & M. cephalus & $\mathrm{KC}, 45^{\circ} 20^{\prime} \mathrm{N}, 36^{\circ} 30^{\prime} \mathrm{E}$ & October 2001 & 11 & $292-495$ \\
\hline L. vanbenedenii & Liza aurata & $\mathrm{KC}, 45^{\circ} 20^{\prime} \mathrm{N}, 36^{\circ} 30^{\prime} \mathrm{E}$ & October 2001 & 9 & $167-275$ \\
\hline L. vanbenedenii & L. aurata & $\mathrm{GV}, 38^{\circ} 11^{\prime} \mathrm{N}, 0^{\circ} 34^{\prime} \mathrm{W}$ & December 2001 & 2 & 107 \\
\hline L. szidati & L. aurata & $\mathrm{KC}, 45^{\circ} 20^{\prime} \mathrm{N}, 36^{\circ} 30^{\prime} \mathrm{E}$ & October 2001 & 3 & 167 \\
\hline
\end{tabular}

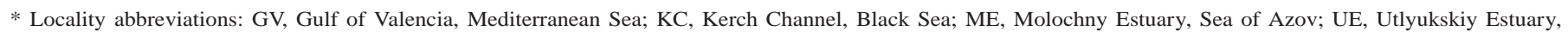
Sea of Azov.

the discriminant analysis. BL and BW were excluded because they may depend on the degree of body contraction and processing technique, and PL and VL were also omitted because they are difficult to measure exactly. The 15 variables were $\log$ transformed and submitted to discriminant analysis using 6 grouping variables: L. pilengas $\mathrm{n}$. $\mathrm{sp}$. from the Black Sea $(\mathrm{n}=9)$, L. pilengas $\mathrm{n}$. sp. from the Sea of Azov $(\mathrm{n}=$ 22), L. chabaudi $(\mathrm{n}=15)$, L. kaohsianghsieni $(\mathrm{n}=14)$, L. mugilinus $(\mathrm{n}=11)$, and $L$. vanbenedenii $(\mathrm{n}=11)$. Because of low sample size, L. szidati was not used as grouping variable, but the 3 specimens belonging to this species were projected over the general pattern obtained with the discriminant analysis. To analyze morphometric differences between $L$. pilengas $\mathrm{n}$. sp. and $L$. chabaudi, Student's $t$-tests were used to establish differences between the means of the 19 morphometric variables (log transformed). Statistical analyses were carried out with SPSS 11.5 for Windows.

Vernacular names of the fish species are according to Froese and Pauly (2003).

\section{RESULTS}

The stepwise discriminant analysis selected 8 of the 15 metric variables as the best discriminating features, namely, VBL, VAA, DAE, DAB, VAE, VAB, VAC, and DAA (listed in decreasing order of importance). The discriminant analysis correctly assigned all specimens to their respective species. Incorrect allocations occurred only between $L$. pilengas $n$. sp. from the Black Sea and from the Sea of Azov: 5 specimens from the Sea of Azov were assigned to the Black Sea and 1 specimen from the Black Sea was assigned to the Sea of Azov. Figure 2 shows the discriminant scores of each specimen plotted against the 2 first discriminant functions (accounting for 84\% of variation between the groups). The specimens of L. pilengas $\mathrm{n}$. $\mathrm{sp}$. can be separated from those of $L$. chabaudi and from those of the rest of the species by their scores along the first discriminant function (Fig. 2). The variables showing high absolute values of standardized coefficients along this function were VAB (0.93), DAA (-0.77), VBL (0.56), VAC (0.53), and VAE $(-0.51)$. Given the position of $L$. pilengas $\mathrm{n}$. sp. specimens along the first function (Fig. 2), such specimens will tend to show larger VAB, VBL, and VAC and lower DAA and VAE relative to those of the other species of Ligophorus. In addition, specimens of $L$. pilengas $\mathrm{n}$. sp. were distinguished from those of $L$. chabaudi by morphometric characters because the $t$-tests showed significant differences $(P<0.05)$ in 18 of the 19 metric characters (all except PAPL) (Table II).

\section{DESCRIPTION}

\section{Ligophorus pilengas $\mathrm{n}$. sp.}

(Figs. 1, 3A, B, 4A)

\section{Synonyms}

Ligophorus vanbenedenii (Parona and Perugia, 1890) sensu Gusev (1985).

Ligophorus chabaudi Euzet and Suriano, 1977, sensu Dmitrieva (1996), Maltsev and Zhdamirov (1996), Maltsev and Miroshnichenko (1998), Domnich and Sarabeev (1999), Domnich and Sarabeev (2000a, 2000b), Sarabeev (2000), and Sarabeev and Domnich (2000).

Worms have the characters of the genus as defined by Euzet and Suriano (1977) and supplemented by Euzet and Sanfilippo (1983). Morphometric measurements of L. pilengas n. sp. specimens mounted in glycerin jelly from the Black Sea and Sea of Azov (this study) are listed in Table II.

Fifteen stained specimens mounted in Canada balsam showed smaller body sizes $(565 \pm 29$ [538-637] long, $87 \pm 7$ [70-94] wide) than the 31 specimens mounted in glycerin jelly (compare with corresponding measurements in Table II). Posterior haptor armed, with 14 hooks, 2 pairs of anchors, 2 transverse bars (Figs. 1D-F, 3A, B). Ventral anchors with elongate thin blade, recurved point. Point short, constituting half the blade length, forming obtuse angle (about $100^{\circ}$ ). Base markedly thicker than blade, separated by notch. Inner root larger than outer root, $\mathrm{VAD} / \mathrm{VAC}=1.36 \pm 0.24(1.13-2.4)$. Angle between roots sharp (about $55^{\circ}$ ). Filament present. Ventral anchors connected by transverse ventral bar. Ventral bar massive, with 2 membranous anterior medial processes (Figs. 1D, 3A). In 13 of 31 specimens (42\%), ventral bar with nonmembranous median process between membranous processes. Morphology of median process highly variable, ranging from small (Figs. 1D, 3A) to massive (Fig. 3B). Dorsal anchors similar in shape to ventral anchors. Inner root much larger than outer roots, DAD/DAC = $1.95 \pm 0.18$ (1.63-2.4), base of dorsal anchors somewhat thinner than that of ventral anchors. Dorsal transversal bar $\mathrm{V}$ shaped, connects dorsal anchors. All 14 marginal hooks subequal with straight handle and sickle (hooklet). Sickle formed by short base with heel, curved blade and filament loop. Male copulatory complex consists of tubular penis about $1 \mu \mathrm{m}$ in 

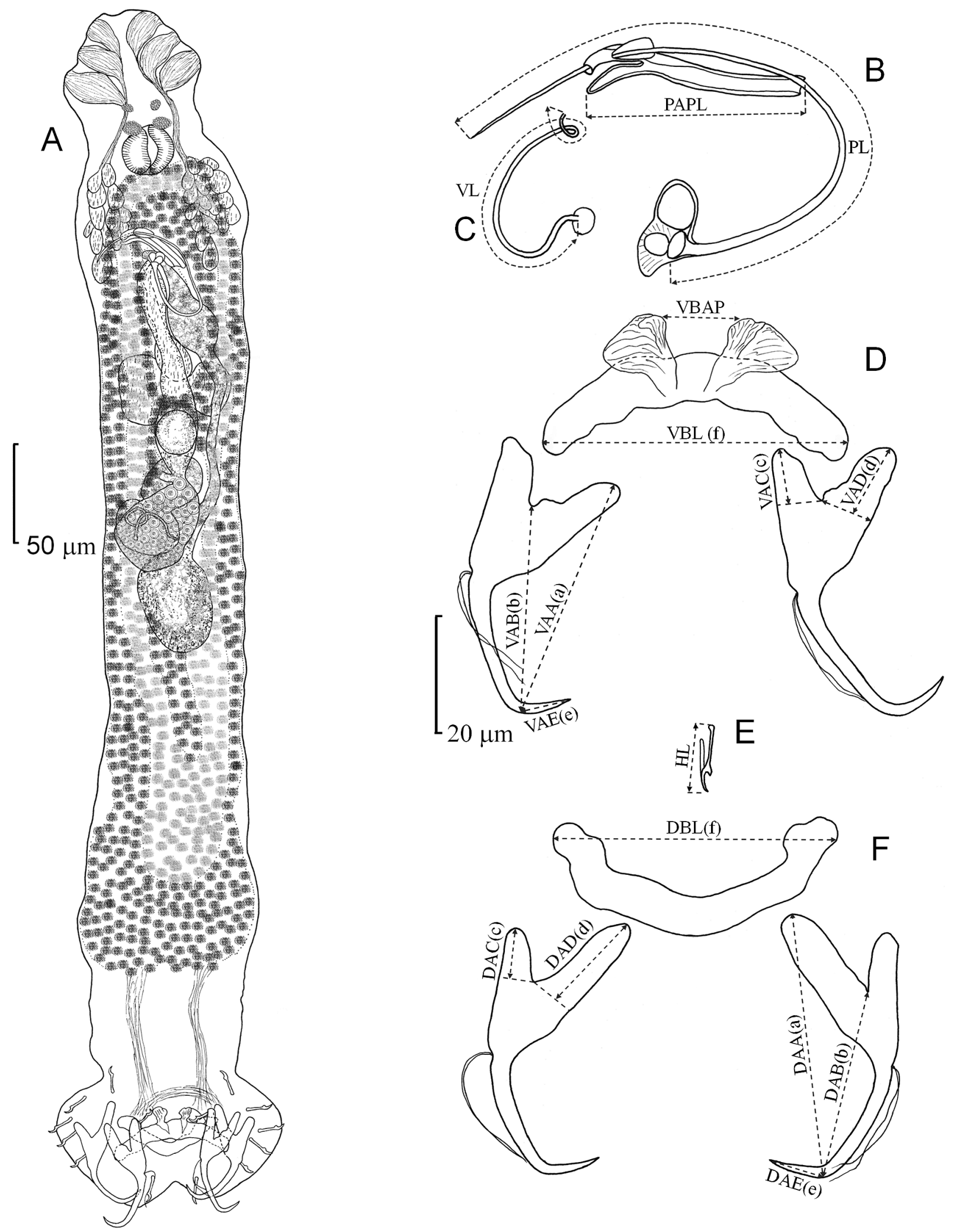

FIGURE 1. Ligophorus pilengas n. sp.: overall view, haptoral and genital sclerotized structures. A. Whole worm, ventral view. B. Male copulatory complex: penis and accessory piece. C. Vaginal armament. D. Ventral bar and anchors. E. Marginal hook. F. Dorsal bar and anchors. For haptoral and genital sclerotized structures, the metric variables studied are shown (see Materials and Methods for abbreviations of measurements). Lowercase letters in parentheses correspond to standard haptor measurements (Murith and Beverley-Burton, 1985). 


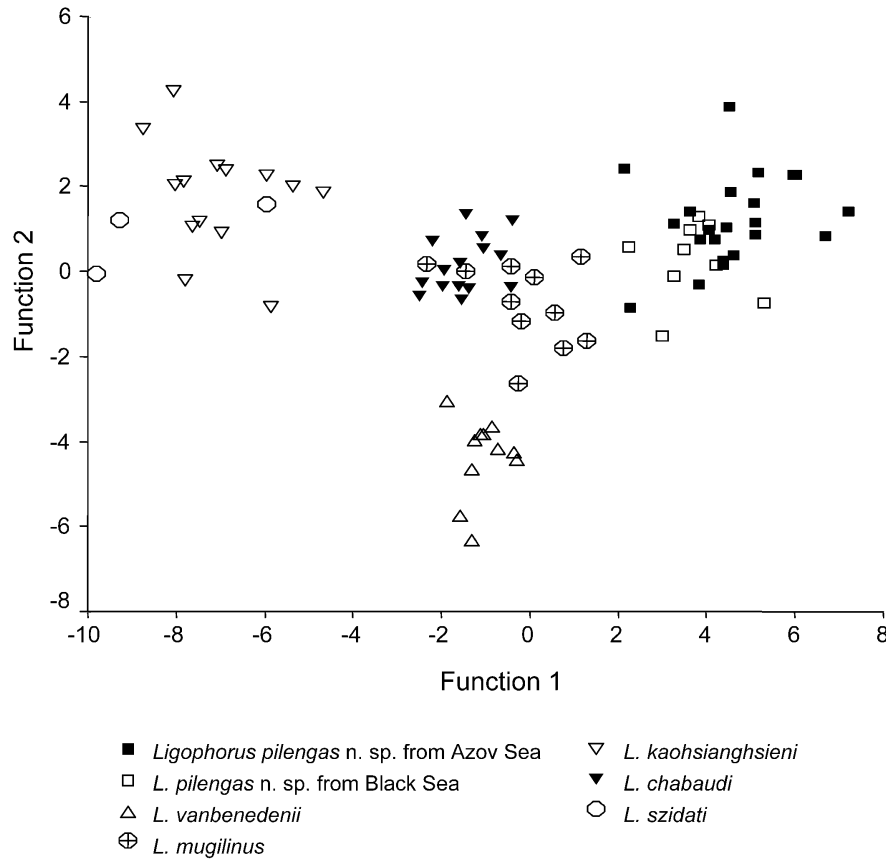

FIGURE 2. Scores resulting from a linear discriminant analysis of metric variables of 85 specimens of Ligophorus (Table I) plotted against the first 2 discriminant functions. diameter and nipper-shaped accessory piece (Figs. 1B, 4A). Distal end of the latter bilobed, lower lobe longer than upper lobe. Accessory piece supports distal end of penis. Vaginal armament is thin, convoluted or straight tube (Fig. 1C).

\section{Taxonomic summary}

Type host: So-iuy mullet, M. soiuy Basilewsky, 1855.

Site of infection: Gill rakers and lamellae.

Type locality: Utlyukskiy Estuary $\left(46^{\circ} 21^{\prime} \mathrm{N}, 35^{\circ} 14^{\prime} \mathrm{E}\right)$, Sea of Azov, Zaporizhzhia Region, Ukraine.

Other localities: Kerch Channel (Black Sea) and Molochny Estuary (Sea of Azov).

Prevalence and intensity range: Prevalence is $87-100 \%$ of adult fish, 2-736 worms per fish.

Specimens deposited: Holotype, 13 paratypes: the Natural History Museum, London (registration numbers BMNH 2003.8.4.1 and BMNH 2003.8.4.13-14).

Etymology: Pilengas is the common name of the type host in Russian and is used as a noun in apposition to form the specific designation of the new species.

\section{Remarks}

Ligophorus pilengas $\mathrm{n}$. $\mathrm{sp}$. is very similar to L. chabaudi, but both species differ from each other in several characters in the morphology of the accessory piece of male copulatory complex and ventral bar. In the distal end of the accessory piece of $L$. pilengas $\mathrm{n}$. sp., the lower lobe is longer than the upper lobe and the basal bifurcation between the lobes is situated at about onethird the distance from the distal end of the accessory piece to the penis (Figs. 1B, 4A). In L. chabaudi, in contrast, the upper

TABLE II. Metrical data of Ligophorus pilengas n. sp. from this study and Dmitrieva's (1996) study compared with measurements of Ligophorus chabaudi from this study. See Materials and Methods for abbreviations of metric variables.

\begin{tabular}{|c|c|c|c|c|c|c|c|c|c|c|c|}
\hline \multirow{3}{*}{$\begin{array}{l}\text { Char- } \\
\text { acters }\end{array}$} & \multicolumn{6}{|c|}{$\begin{array}{l}\text { This study, } \\
\text { Ligophorus pilengas } \mathrm{n} . \mathrm{sp} .\end{array}$} & \multicolumn{2}{|c|}{$\begin{array}{l}\text { Dmitrieva (1996), } \\
\text { L. pilengas } \mathrm{n} . \mathrm{sp} . \\
(=\text { L. chabaudi) }\end{array}$} & \multirow{2}{*}{\multicolumn{3}{|c|}{$\begin{array}{c}\text { This study, } \\
\text { L. chabaudi } \\
\begin{array}{c}\text { Black Sea } \\
(\mathrm{n}=15)\end{array}\end{array}$}} \\
\hline & \multicolumn{3}{|c|}{$\begin{array}{l}\text { Black Sea } \\
(\mathrm{n}=9)\end{array}$} & \multicolumn{3}{|c|}{$\begin{array}{l}\text { Sea of Azov } \\
\quad(\mathrm{n}=22)\end{array}$} & \multirow{2}{*}{$\begin{array}{c}\text { Black Sea } \\
\text { Range }\end{array}$} & \multirow{2}{*}{$\begin{array}{c}\text { Pacific } \\
\text { Ocean } \\
\text { Range }\end{array}$} & & & \\
\hline & Mean & SD & Range & Mean & SD & Range & & & Mean & SD & Range \\
\hline VAA & 41 & 2 & $37-44$ & 44 & 3 & $38-49$ & $45-49$ & $41-44$ & 36 & 1 & $33-38$ \\
\hline VAB & 33 & 2 & $29-35$ & 35 & 3 & $30-43$ & $37-41$ & $31-37$ & 27 & 2 & $24-30$ \\
\hline VAC & 11 & 1 & $9-13$ & 12 & 2 & $9-14$ & $10-12$ & $9-12$ & 10 & 1 & $8-12$ \\
\hline VAD & 15 & 2 & $13-19$ & 16 & 2 & $13-20$ & $12-16$ & $11-16$ & 17 & 1 & $15-18$ \\
\hline VAE & 9 & 0.5 & 8-9 & 9 & 0.4 & $9-10$ & $9-10$ & $9-10$ & 10 & 1 & $9-12$ \\
\hline DAA & 40 & 2 & $35-44$ & 46 & 3 & $39-50$ & $46-50$ & $43-47$ & 37 & 2 & $34-39$ \\
\hline DAB & 30 & 2 & $27-32$ & 32 & 2 & $27-37$ & $33-36$ & $31-34$ & 29 & 1 & $27-31$ \\
\hline VBL & 48 & 1 & $46-50$ & 52 & 5 & $46-68$ & $50-59$ & $43-54$ & 38 & 2 & $36-41$ \\
\hline VBAP & 9 & 1 & $9-10$ & 11 & 2 & $7-14$ & - & - & 5 & 1 & $3-7$ \\
\hline DBL & 45 & 4 & $40-55$ & 49 & 4 & $43-62$ & $47-61$ & $39-50$ & 36 & 3 & $31-41$ \\
\hline PAPL & 36 & 3 & $32-41$ & 38 & 3 & $29-43$ & - & - & 35 & 3 & $28-38$ \\
\hline PL & 95 & 7 & $89-111$ & 95 & 6 & $83-107$ & $118-130$ & $116-122$ & 90 & 6 & $79-102$ \\
\hline VL & 36 & 11 & $22-60$ & 30 & 9 & $14-51$ & $56-59$ & $56-74$ & 43 & 12 & $28-70$ \\
\hline
\end{tabular}



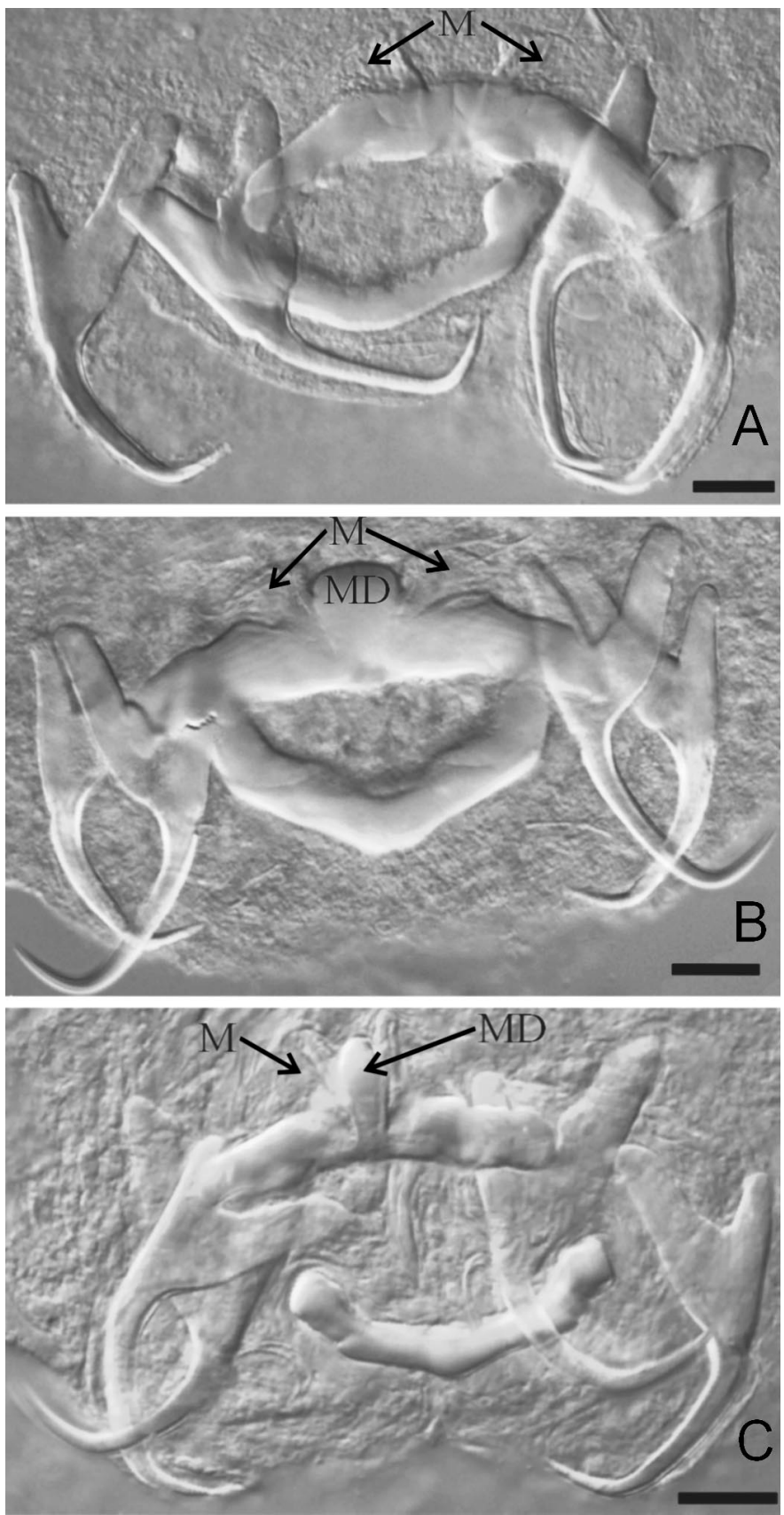

FIGURE 3. Photomicrographs of sclerotized elements of haptors of Ligophorus pilengas $\mathrm{n}$. sp. and Ligophorus chabaudi. A-B. Sclerotized elements of $L$. pilengas $\mathrm{n}$. sp. haptors, showing different types of ventral bar with membranous (M) and median (MD) anterior processes. C. Sclerotized elements of a $L$. chabaudi haptor. Bar $=10 \mu \mathrm{m}$.

lobe is longer than the lower lobe, and the basal bifurcation between them is at the middle of the accessory piece (Fig. 4B, see also original illustration of Euzet and Suriano [1977] and redrawing by Dmitrieva and Gerasev [1996]). In addition, the membranous anterior processes of the ventral bar of $L$. pilengas n. sp. are distinctly separated from the median process (if the latter is present) (Figs. 1D, 3A, B), whereas the membranous processes are contiguous to the median process in $L$. chabaudi (Fig. 3C). Furthermore, L. pilengas n. sp. differs significantly from L. chabaudi in 18 metric measurements. In L. pilengas $\mathrm{n}$ sp., the mean values of BL, BW, VAD, VAE, and VL are significantly smaller and those of VAA, VAB, VAC, DAA, DAB, DAC, DAD, DAE, HL, VBL, VBAP, DBL, and PL are significantly larger than in $L$. chabaudi.

Ligophorus pilengas $\mathrm{n}$. sp. differs from L. szidati, L. confusus, L. angustus, L. leporinus, L. chongmingensis, and L. kaohsianghsieni in most morphometric traits (Euzet and Suriano, 1977; Zhang and Ji, 1981; Gusev, 1985; Hu and Li, 1992; Yang, 2001). Therefore, this discussion is restricted to the remainder of species of Ligophorus. The new species is similar to L. parvicirrus, L. macrocolpos, L. acuminatus, and L. chenzhenensis in the shape and measurements of anchors and, additionally, to L. macrocolpos and L. acuminatus in ventral bar morphology. However, the new species differs from $L$. parvicirrus in the morphology and size of both the ventral bar and the male copulatory complex (Euzet and Sanfilippo, 1983) and from L. chenzhenensis in the morphology of the ventral bar, the upper lobe of the accessory piece of the male copulatory complex, and the distal end of the vaginal armament, as well as in the length of the penis and the body ( $\mathrm{Hu}$ and $\mathrm{Li}, 1992)$. Ligophorus pilengas n. sp. differs from L. macrocolpos and L. acuminatus in the shape and smaller length of the accessory piece of the male copulatory complex (Euzet and Suriano, 1977). The shape of the anchors and ventral bar of L. pilengas $\mathrm{n}$. sp. is similar to that of L. vanbenedenii, L. mugilinus, L. minimus, L. imitans, L. heteronchus, L. euzeti, L. hamulosus, and L. huitrempe, but the new species differs from $L$. vanbenedenii and L. hamulosus because in these species all metrical characters are smaller (Euzet and Suriano, 1977; Pan, 1999) and from L. mugilinus because the total and main part of the anchors and the ventral bar are smaller than in the new species (Euzet and Suriano, 1977). Likewise, the outer root and point of L. heteronchus and $L$. euzeti are smaller than in L. pilengas n. sp. (Euzet and Suriano, 1977; Dmitrieva and Gerasev, 1996). In addition, L. heteronchus has a smaller inner root and ventral bar than in the new species (Euzet and Suriano, 1977). Finally, the outer root and ventral bar of $L$. imitans and $L$. minimus and the main part of the anchors and ventral bar of L. huitrempe are smaller than in L. pilengas $\mathrm{n}$. sp. (Euzet and Suriano, 1977; Fernández, 1987). The new species can also be distinguished from the latter 8 species by the shape of the accessory piece of the male copulatory complex and also from $L$. vanbenedenii, L. mugilinus, $L$. minimus, L. heteronchus, L. hamulosus, and L. huitrempe because the accessory piece is shorter in these species.

\section{DISCUSSION}

Both the morphological and metric differences between $L$. pilengas $\mathrm{n}$. sp. from the So-iuy mullet and those of other species of Ligophorus suggest that for the So-iuy mullet specimens represent a new species. Gusev (1985) studied specimens of Ligophorus on So-iuy mullets from the Liao-Ho River (Yellow Sea basin), which he tentatively identified as L. vanbenedenii. Although he did not provide body measurements of the specimens, judging from the drawings of the haptoral sclerotized elements, we think that Gusev's (1985) specimens correspond to L. pilengas n. sp. In particular, the ventral bar of Gusev's (1985) specimens appear to have membranous processes similar to $L$. pilengas $\mathrm{n}$. sp. (membranous processes are absent in $L$. 

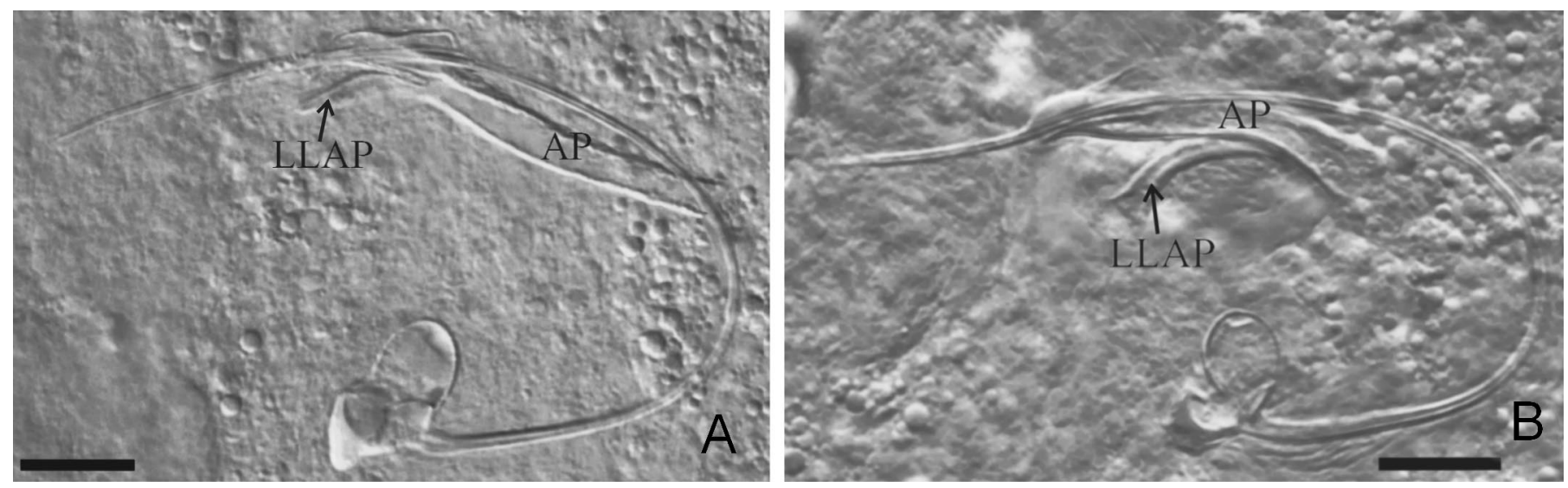

FigURE 4. Photomicrographs of sclerotized elements of male copulatory complex of Ligophorus pilengas n. sp. and Ligophorus chabaudi. A. Sclerotized elements of $L$. pilengas $\mathrm{n}$. sp. male copulatory complex, showing the accessory piece (AP) and lower lobe of the accessory piece (LLAP). B. Sclerotized elements of $L$. chabaudi male copulatory complex. Bar $=10 \mu \mathrm{m}$.

vanbenedenii), and the shape of the accessory piece conforms to that of L. pilengas n. sp. Likewise, the specimens from the So-iuy mullet in the Black Sea Basin and the western Pacific identified as L. chabaudi by Dmitireva (1996) should be assigned to $L$. pilengas $\mathrm{n}$. sp. because the ventral bar lacks the median process, the copulatory complex is like that of $L . p i$ lengas n. sp., and the metric data seem closer to those of $L$. pilengas $\mathrm{n}$. sp. than to those of L. chabaudi (Table II). After examination of the original material, we also conclude that other monogenean specimens reported from the So-iuy mullet and identified as L. chabaudi in different studies (Maltsev and Zhdamirov, 1996; Maltsev and Miroshnichenko, 1998; Domnich and Sarabeev, 1999, 2000a, 2000b; Sarabeev, 2000; Sarabeev and Domnich, 2000) are actually L. pilengas n. sp.

This new species is the second member of Ligophorus recorded in the So-iuy mullet (the first being L. kaohsianghsieni). The So-iuy mullet was introduced in the late 1970s into the Black Sea and Sea of Azov from the western Pacific. Considering previous records of $L$. pilengas $\mathrm{n}$. sp. in the western $\mathrm{Pa}-$ cific (Gusev, 1985; Dmitrieva, 1996), it is probable that this parasite was brought to the Black Sea and Sea of Azov together with its host.

As in most monogeneans, the taxonomy of Ligophorus relies on the shape of the sclerotized structures, which are used for species characterization (Euzet and Suriano, 1977; Euzet and Sanfilippo, 1983; Gusev, 1985; Fernández, 1987; Dmitrieva and Gerasev, 1996; Pan, 1999). Statistical classifiers based on these characters might be useful for classification of monogenean species, as shown for species of Gyrodactylus (Shinn et al., 2000). In fact, the results of our discriminant analysis give support to this contention because it correctly assigned all specimens to their respective species of Ligophorus. The analysis also showed that the form from the So-iuy mullet could be unequivocally and objectively distinguished from 5 species of Ligophorus known from hosts that co-occur with M. soiuy in the basin of the Black Sea, thus supporting that this form represents a new species. The discriminant analysis suggests that L. pilengas $\mathrm{n}$. $\mathrm{sp}$. can be characterized mostly by measurements of the main part of the ventral anchors and ventral bar.

However, the morphology of sclerotized structures should be used with caution in the taxonomy of the ancyrocephalids be- cause of both potential intraspecific variability (Ferdig et al., 1991) and dependence of metric characters on host size (Caltran et al., 1995). In L. pilengas n. sp., we observed variability in the morphology of the ventral bar, concerning mostly the occurrence and shape of a medial process, and the discriminant analysis revealed morphometric differences between the specimens from the Sea of Azov and the Black Sea. However, these morphometric variations appeared smaller than interspecific differences and might be attributable to geographical differences in development, as proposed for other ancyrocephalids (Ferdig et al., 1991). As for the influence of host size, most specimens of L. pilengas $\mathrm{n}$. sp. were from hosts of similar body length as those of L. chabaudi (Table I). Therefore, the morphometric differences between these 2 forms cannot be attributed to this factor.

The diagnosis of Ligophorus by Euzet and Suriano (1977) and supplemented by Euzet and Sanfilippo (1983) conforms to the characters present in L. pilengas n. sp. However, the comparison between $L$. pilengas $\mathrm{n}$. sp. and $L$. chabaudi provided in this study suggests that the following feature should be considered in a revised generic diagnosis: ventral transversal bar can have 2 membranous anterior processes flanking and a nonmembranous median process between them.

\section{ACKNOWLEDGMENTS}

We thank Aneta Kostadinova, Bulgarian Academy of Sciences, for valuable criticism and suggestions, and Vyacheslav Maltsev, Southern Scientific Research Institute of Marine Fisheries and Oceanography (Kerch, Ukraine), for his help in collecting some of the material. V.L.S. benefited a Young Scientist Fellowship (YSF 01/1-0203) from INTAS and a North Atlantic Treaty Organization Grant for Young Scientists (71/B/02/SP) from the Ministry of Science and Technology of Spain. Funds for this study were partially provided by The Ministry of Education and Science of Ukraine.

\section{LITERATURE CITED}

Caillot, C., S. Morand, C. M. Muller-Graf, E. Faliex, and B. Marchand. 1999. Parasites of Dicentrarchus labrax, Anguilla an guilla and Mugil cephalus from a pond in Corsica, France. Journal of the Helminthological Society of Washington 66: 95-98.

Caltran, H., P. Silan, And M. Roux. 1995. Ligophorus imitans (Monogenea) ectoparasite de Liza ramada (Teleostei). II. Variabilité mor- 
phologique et contraintes environnementales. Ecologie 26: 105113.

Dmitrieva, E. V. 1996. Fauna of Monogenea of the Far-East Mugil soiuy in the Black Sea. Vestnik Zoologii 4/5: 95-97. [In Russian.]

_ AND P. I. GERASEV. 1996. Monogenean of the genus Ligophorus (Ancyrocephalidae)_-Parasites of the Black Sea mullets (Mugilidae). Parazitologiya 30: 440-449. [In Russian.]

Domnich, I. F., AND V. L. SARABEEV. 1999. Formation of the parasite fauna of Mugil soiuy in the Azov Sea. Visnyk Zaporizkogo Derzhavnogo Universytetu 2: 218-223. [In Ukrainian.]

, AND - 2000a. Forming of the parasitic fauna of the pilengas in the Azov Sea. Acta Parasitologica 45: 265.

, AND $-2000 \mathrm{~b}$. Parasitic fauna structure of the pilengas in the Azov Sea. Acta Parasitologica 45: 268.

EuZET, L., AND D. SANFILIPPO. 1983. Ligophorus parvicirrus n. sp. (Monogenea, Ancyrocephalidae) parasite de Liza ramada (Risso, 1826) (Teleostei, Mugilidae). Annales de Parasitologie Humaine et Comparée 58: 325-335.

—, AND D. M. SURIANO. 1977. Ligophorus n. g. (Monogenea, Ancyrocephalidae) parasite des Mugilidae (Téléostéens) en Méditerranée. Bulletin du Muséum National d'Histoire Naturelle, 3e Série, Zoologie 472: 799-821.

Ferdig, M. T., M. A. McDowell, ANd J. Janovy JR. 1991. Salsuginus yutanensis n. sp. (Monogenea: Ancyrocephalidae) from Fundulus sciadicus in Clear Creek of eastern Nebraska. Journal of Parasitology 77: $58-61$.

FERNÁNDEZ, J. 1987. Los parásitos de la lisa Mugil cephalus L., en Chile: Sistemática y aspectos poblacionales (Perciformes: Mugilidae). Gayana Zoológica 51: 3-58.

Froese, R., AND D. PaUly. 2003. FishBase. Available at: www.fishbase. org. Accessed 26 February 2003.

Gusev, A. V. 1983. Methods for collection and preparation of monogeneans parasitizing fish. Nauka, Leningrad, U.S.S.R., 46 p. [In Russian.]

- 1985. Order Dactylogyridea. In Keys to the parasites of freshwater fish of the USSR fauna. Metazoan parasites, vol. 2, A. V Gusev, M. N. Dubinina, O. N. Pugachev, E. V. Raykova, I. A Hotenovskiy, and R. Ergens (eds.). Nauka, Leningrad, U.S.S.R., p. 15-251. [In Russian.]

Hu, Z., AND D. Li. 1992. Two new species of monogenetic trematodes of marine fishes Mugil cephalus from the Chongming Island, Shanghai, China. Journal of Shanghai Teachers University (Natural Sciences) 21: 67-70. [In Chinese.]

Maltsev, V. N., AND A. I. MiroshnichenKo. 1998. Peculiarities of monogenean infestation of the Pacific haarder in the Azov Sea. In
Problems in flatworm systematics and phylogeny. Zoological Institute of the Russian Academy of Sciences, Saint Petersburg, Russia, p. 66-67. [In Russian.]

, AND V. N. ZhDAMIROV. 1996. Parasitofauna of the mullet haarder (Mugil soiuy Basilewsky) of the Kerch channel. Proceedings of the Southern Scientific Research Institute of Marine Fisheries and Oceanography 44: 229-232. [In Russian.]

Miroshnichenko, A. I., AND V. N. Maltsev. 1998. Monogenean fauna of the golden grey and striped mullet in the Azov Sea. In Problems in flatworm systematics and phylogeny. Zoological Institute of the Russian Academy of Sciences, Saint Petersburg, Russia, p. 68-69. [In Russian.]

Murith, D., AND M. Beverley-Burton. 1985. Salsuginus BeverleyBurton, 1984 (Monogenea, Ancyrocephalidae) from Cyprinodontoidei (Atheriniformes) in North-America with descriptions of Salsuginus angularis (Mueller, 1934) Beverley-Burton, 1984 from Fundulus diaphanus and Salsuginus heterocliti n. sp. from Fundulus heteroclitus. Canadian Journal of Zoology 63: 703-714.

PAN, J. 1999. Monogenea of marine fishes from Hainan Island V. One new species of the genus Ligophorus from the South China Sea. Zoological Research 20: 186-188. [In Chinese.]

Radujković, B. M., AND A. RaIbaut. 1989. Parasites des poissons marins du Monténégro: Liste des espèces de poissons avec leurs parasites. Acta Adriatica 30: 307-319.

SARABEEV, V. L. 2000. Parasites of the pilengas and local fish species in the northwestern part of the Azov Sea (fauna, ecology). Abstract of Ph.D. Thesis. Schmalhausen Institute of Zoology, NASU, Kiev, Ukraine, 20 p. [In Ukrainian.]

, AND I. F. Domnich. 2000. Age-related dynamics of parasitic infection of the pelingas (Mugil soiuy) in the Molochny estuary of the Azov Sea. Vestnik Zoologii, Supplement 14: 6-12. [In Russian.]

Shinn, A. P., J. W. Kay, AND C. Sommerville. 2000. The use of statistical classifiers for the discrimination of species of the genus $G y$ rodactylus (Monogenea) parasitizing salmonids. Parasitology 120: 261-269.

YANG, T. B. 2001. Ligophorus ellochelon sp. nov. In Monogeneans of Chinese marine fishes, J. Y. Zhang, T. B. Yang and L. Liu (eds.). Agriculture Press, Beijing, People's Republic of China, p. 169-171. [In Chinese.]

ZhANG, J., AND G. Ji. 1981. Monogenetic trematodes of Chinese marine fishes: Two species of Ancyrocephalus from the gills of Mugil cephalus, with description of a new species. Oceanologia et Limnologia Sinica 12: 349-353. [In Chinese.]

, T. YANG, L. LiU, AND X. Ding. 2003. A list of monogeneans from Chinese marine fishes. Systematic Parasitology 54: 111-130. 